\title{
Quasielastic neutron scattering experiments including activation energies and mathematical modeling of methyl halide dynamics
}

O. Kirstein, M. Prager, H. Grimm, A. Buchsteiner, and A. Wischnewski

Citation: The Journal of Chemical Physics 127, 094504 (2007); doi: 10.1063/1.2760205

View online: https://doi.org/10.1063/1.2760205

View Table of Contents: http://aip.scitation.org/toc/jcp/127/9

Published by the American Institute of Physics

\section{Articles you may be interested in}

A time-of-flight backscattering spectrometer at the Spallation Neutron Source, BASIS

Review of Scientific Instruments 82, 085109 (2011); 10.1063/1.3626214

Quasielastic and inelastic neutron scattering study of methyl group rotation in solid and liquid pentafluoroanisole and pentafluorotoluene

The Journal of Chemical Physics 128, 194502 (2008); 10.1063/1.2894847

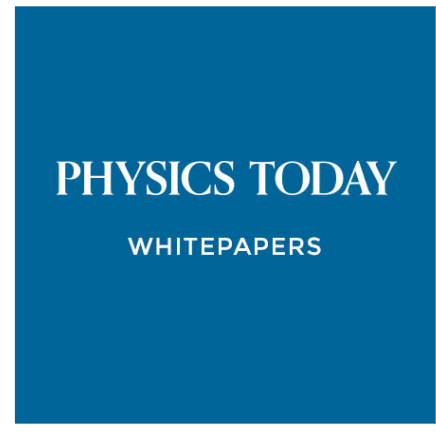




\title{
Quasielastic neutron scattering experiments including activation energies and mathematical modeling of methyl halide dynamics
}

\author{
O. Kirstein ${ }^{\mathrm{a})}$ \\ Bragg Institute, Australian Nuclear Science and Technology Organisation, Menai, New South Wales 2234, \\ Australia \\ M. Prager and H. Grimm \\ Institut für Festkörperforschung, Forschungszentrum Jülich GmbH, D-52425 Jülich, Germany
}

A. Buchsteiner

Berlin Neutron Scattering Center, Hahn-Meitner Institut, D-14109 Berlin, Germany

A. Wischnewski

Institut für Festkörperforschung, Forschungszentrum Jülich GmbH, D-52425 Jülich, Germany

(Received 24 January 2007; accepted 26 June 2007; published online 4 September 2007)

\begin{abstract}
Quasielastic neutron scattering experiments were carried out using the multichopper time-of-flight spectrometer V3 at the Hahn-Meitner Institut, Germany and the backscattering spectrometer at Forschungszentrum Jülich, Germany. Activation energies for $\mathrm{CH}_{3} \mathrm{X}, \mathrm{X}=\mathrm{F}, \mathrm{Cl}, \mathrm{Br}$, and $\mathrm{I}$, were obtained. In combination with results from previous inelastic neutron scattering experiments the data were taken to describe the dynamics of the halides in terms of two different models, the single particle model and the coupling model. Coupled motions of methyl groups seem to explain the dynamics of the methyl fluoride and chloride; however, the coupling vanishes with the increase of the mass of the halide atom in $\mathrm{CH}_{3} \mathrm{Br}$ and $\mathrm{CH}_{3}$ I. (C) 2007 American Institute of Physics.
\end{abstract}

[DOI: 10.1063/1.2760205]

\section{INTRODUCTION}

The methyl halides belong to a simple class of molecules that contain $\mathrm{CH}_{3}$ groups. Systematic experimental studies of the quantum rotation of the methyl group in $\mathrm{CH}_{3} \mathrm{~F},-\mathrm{Cl},-\mathrm{Br}$, and $-\mathrm{I}$ can be found in the literature. ${ }^{1,2}$ The crystal structures of $\mathrm{CH}_{3} \mathrm{Br},-\mathrm{Cl}$, and $-\mathrm{I}$ have been known for almost 20 years, ${ }^{3-6}$ whereas the structure of deuterated fluoride was solved in 1996 by high-resolution neutron powder diffraction using time-of-flight techniques. ${ }^{7}$

Usually, the dynamics of the majority of rotating $\mathrm{CH}_{3}$ groups in molecular crystals can be described by a meanfield model known as the single particle model (SPM). ${ }^{8}$ An analysis of experimental results which goes beyond a phenomenological use of the SPM must be able to explain the rotational potential, which determines the dynamics of the rotating group based on the crystal structure and the fundamental interatomic and intermolecular interactions. There are different ways of modeling the intermolecular interactions and thereby the dynamics of the molecules (for example, by using density functional theory, quantum chemistry, or transferable pair-potential calculations). The latter method was used to describe the lattice and rotational dynamics of the methyl halides by using pair potentials based on universal force fields, ${ }^{9,10}$ and values that characterize the potential were calculated and compared to earlier experimental results. In order to bring a comprehensive understanding to the experiments done so far, quasielastic neutron scattering experi-

\footnotetext{
a) Author to whom correspondence should be addressed. Fax: +61-2 97173606; Electronic mail: oliver.kirstein@ansto.gov.au
}

ments were performed to obtain values for the activation energies, which were then compared to the calculations and used to refine potential parameters.

\section{THEORY}

\section{A. Single particle model}

The SPM describes the dynamics of many molecular crystals that contain either $\mathrm{CH}_{3}, \mathrm{NH}_{3}$, or $\mathrm{NH}_{4}$ groups reasonably well and is outlined briefly below. In this model it is assumed that an isolated group is hindered to reorient because of its interaction with its environment. For a $\mathrm{CH}_{3}$ group the reorientation typically occurs around the bond that links the carbon to the rest of the molecule and thus defines the axis of rotation. The rotational potential is then expressed as a function of a single rotational coordinate. The potential depends on the symmetry of the rotating unit $\left(C_{3 v}\right.$ for a methyl group) and its environment and is usually expressed as the sum of the first two terms of a Fourier expansion,

$$
V(\varphi)=V_{0}+\sum_{n=1}^{2} \frac{V_{3 n}}{2}\left(1-\cos \left(3 n \varphi+\alpha_{3 n}\right)\right)
$$

The rotational excitations are determined by the potential given in Eq. (1). Excitations and transitions to be compared to, e.g., a neutron scattering experiment are obtained by solving the Schrödinger equation, 


$$
\begin{aligned}
& H\left|\Psi_{i}\right\rangle=E_{i}\left|\Psi_{i}\right\rangle, \\
& {\left[-B \frac{d^{2}}{d \varphi^{2}}+V(\varphi)\right] \Psi_{i}=E_{i} \Psi_{i},}
\end{aligned}
$$

where $B=0.655 \mathrm{meV}$ is the rotational constant for a methyl group. In order to obtain the energy levels it is sufficient to consider space coordinates. The space wave functions are expanded into free rotor functions $|m\rangle=\exp (\operatorname{im} \varphi)$ according to $\Psi_{i}=\Sigma_{m} \alpha_{m}|m\rangle$, and the eigenvalues of the Hamiltonian matrix $H_{m n}$ are calculated by applying standard numerical procedures. Parameters of rotational potentials can then be derived from experimentally determined transition energies.

\section{B. Coupling model}

In a different approach to explain the observed transitions a simple coupling model (CM) assumes two methyl groups which share a common plane. ${ }^{11}$ The Hamiltonian that describes the interaction between two in-plane methyl groups is given by

$$
H=H_{1}+H_{2}+W_{12},
$$

with single particle terms,

$$
H_{i}=-B \frac{\partial^{2}}{\partial \varphi_{i}^{2}}+\frac{V_{3 i}}{2}\left(1-\cos 3 \varphi_{i}\right), \quad i=1,2 .
$$

Given the above geometry, the coupling depends on the differences in orientations only and $W_{12}$ can be expressed as

$$
W_{12}=W_{3} \cos \left(3 \varphi_{1}-3 \varphi_{2}\right) \text {. }
$$

Calculations show ${ }^{11}$ that for crystallographically equivalent and identical particles, $V_{3}=V_{31}=V_{32}$, the librational ground state consists of four tunneling sublevels labeled by the symmetry of the tunneling states of each of the two $\mathrm{CH}_{3}$ groups and transitions are possible for $A A \rightarrow A E, A A \rightarrow E_{a} E_{b}, A A$ $\rightarrow E_{a} E_{a}$, and $E_{a} E_{a} \rightarrow E_{a} E_{b}$. Within the CM the activation energy is calculated via the distance between the ground state and the saddle point of the potential surface $V\left(\varphi_{1}, \varphi_{2}\right)$.

\section{Scattering function for quasielastic scattering}

Reorientations can occur when the single particle has enough thermal energy to hop over the barrier. The scattering function $S(Q, \omega)$ for a powder sample with protons jumping between three equivalent sites on a circle with a jump frequency $\nu$ is given by

$$
S(Q, \omega)=A_{0}(Q) \delta(\omega)+A_{1}(Q) \frac{1}{\pi} \frac{(3 / 2) \nu}{(3 / 2 \nu)^{2}+\omega^{2}},
$$

with prefactors $A_{0}(Q)=\frac{1}{3}\left(1+2 j_{0}(Q d)\right)$ and $A_{1}(Q)=\frac{2}{3}(1$ $\left.-j_{0}(Q d)\right) .{ }^{12} Q$ is the value of the scattering vector and $d$ the jump length, i.e., the proton-proton distance of $d=1.78 \AA$ inside the $\mathrm{CH}_{3}$ group. The width of the Lorentzian in Eq. (6) is given by $\Gamma / \hbar=(3 / 2) \nu$. Since the proton jumps are thermally activated processes the jump frequency $\nu$ and therefore the linewidth $\Gamma$ of the Lorentzian depend on the temperature and show an Arrhenius behavior,

$$
\Gamma(T)=\Gamma_{0} \exp \left(-\frac{E_{a}}{k_{B} T}\right) .
$$

Equation (7) can be used to determine the activation energy $E_{a}$, which is the energy difference between the ground state and the maximum of the methyl rotational potential.

\section{EXPERIMENT}

\section{A. Instruments}

Experiments were carried out using the multichopper time-of-flight instrument V3 [known also as NEAT (Ref. 13)] at the Berlin Centre for Neutron Scattering (BENSC), HahnMeitner Institut (HMI) in Berlin, Germany, as well as the high-resolution backscattering spectrometer BSJ (Ref. 14) at the FRJ2 reactor at the Forschungszentrum Jülich, Germany. NEAT was configured with an incident wavelength of $\lambda$ $=8 \AA$ with a corresponding energy resolution of $\delta E$ $=30 \mu \mathrm{eV}$. The spectrometer BSJ was used in the symmetric configuration with the standard silicon monochromator giving an incident wavelength of $\lambda=6.27 \AA$. This yields an energy transfer range of $\pm 17 \mu \mathrm{eV}$ around the incident energy of $E_{i}=2.08 \mathrm{meV}$ with an energy resolution $\delta E=1.0 \mu \mathrm{eV}$. On both instruments the sample temperature was controlled in a standard Orange cryostat. The overall temperature range was $44 \mathrm{~K} \leqslant T \leqslant 169 \mathrm{~K}$ and adjusted accordingly depending on the respective sample under investigation.

\section{B. Sample preparation}

The samples were obtained commercially from Aldrich with a chemical purity $\geqslant 99 \%$ and were used in all experiments without any further purification. Gaseous samples were condensed into the sample container with dimensions of $35 \times 40 \times 0.5 \mathrm{~mm}^{3}$. After liquefaction the samples were cooled down into the solid phase. The scattering probabilities of the samples were approximately $20 \%$. The standard aluminum sample container was positioned under an angle of $60^{\circ}$ to the incident beam to favor scattering at higher angles and minimize the effect of multiple scattering.

\section{Data processing}

The experimental data obtained were transformed into $S_{\text {exp }}(Q, \omega)$ using standard programs ${ }^{15}$ in order to derive the relevant physical parameters. The data manipulation involved the following steps: subtraction of the background, determination of the resolution function, convolution of the theoretical spectrum with the resolution function, fit of the convoluted spectra to the measured data to obtain the linewidth of $S_{\text {exp }}(Q, \omega)$, and correction for multiple scattering. The formalism used to correct for multiple scattering follows the procedure described in Ref. 16. It is assumed that the fraction $s$ of neutrons scattered $n+1$ times to those scattered $n$ times does not depend on $n$ and neutrons that are scattered twice are distributed isotropically.

The experimentally determined scattering function $S_{\exp }(Q, \omega)$ is then given by 


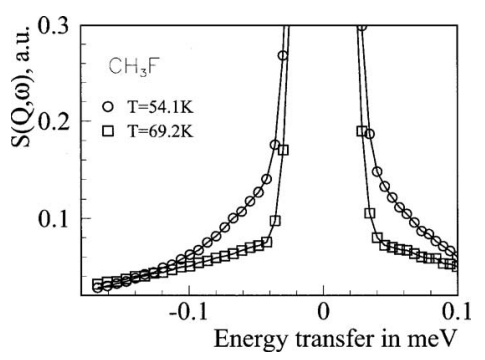

FIG. 1. Quasielastic spectra of $\mathrm{CH}_{3} \mathrm{~F}$ obtained using the NEAT spectrometer at HMI.

$$
\begin{aligned}
S_{\exp }(Q, \omega)= & S_{0}(Q, \omega)+s \cdot \overline{S(\omega)} \otimes \overline{S(\omega)}+s^{2} \cdot \overline{S(\omega)} \\
& \otimes \overline{S(\omega)} \otimes \overline{S(\omega)}+\cdots+,
\end{aligned}
$$

where $\otimes$ represents the convolution of the angular averaged true scattering function $\overline{S(\omega)}$ with itself in $\omega$, effectively smearing out or broadening the true scattering function $S_{0}(Q, \omega)$. This means for the Arrhenius plot, in which the logarithm of the linewidth is plotted against $1 / T$ in order to derive the activation energy, that the expected "straight" line is shifted toward lower logarithms of the linewidth. Consequently, for a given $Q$ value the attempt frequency $\Gamma_{0}$ is shifted to lower values as well. Although the procedure is $Q$ dependent it influences $S_{\text {exp }}(Q, \omega)$ more significantly for low $Q$ values since $\overline{S(\omega)}$ is dominated by the strong $Q$ dependence of Eq. (6) due to the angular averaging. For our purpose we are mainly interested in determining $S_{\exp }(Q, \omega)$ at large values of $Q$ to take advantage of the large amplitude $A_{1}(Q)$ in Eq.(6) and are therefore less sensitive to the effect of multiple scattering despite the $20 \%$ scattering probability.

\section{Potential parameter determination}

The relevant model parameters were determined by using different subroutines provided by the CERN library, namely, MINSQ and LEAMAX (Ref. 17) with a user-defined function in which the model transitions are functions of the unknown potential parameters $V_{3}, V_{6}$, and $\alpha_{6}$ in the case of the SPM and $V_{3}$ and $W_{3}$ in the case of the CM. Function minimization and least-square fits use Levenberg-Marquardt algorithms to determine an individual $\chi_{\exp }^{2}$ for each molecule to get an estimate of how good the model actually reproduces the experimental data. In the case of the coupling model three experimental transitions are used to determine two potential parameters resulting in a fit with one parameter of freedom, $\nu=1$. Calculating the goodness-of-fit probability,

$$
Q\left(\chi_{\exp }^{2}, \nu\right)=\int_{\chi_{\exp }^{2}}^{\infty} P\left(\chi_{\exp }^{2}, \nu\right) d \chi_{\exp }^{2},
$$

allows us to accept or reject the hypothesis that the CM is the appropriate model when the probability $Q$ indicates that $\chi_{\exp }^{2}$ is likely to have occurred. ${ }^{18}$

\section{RESULTS}

\section{A. Quasielastic line broadening}

For illustration purposes two of the spectra obtained for the $\mathrm{CH}_{3} \mathrm{~F}$ using the NEAT spectrometer are shown in Fig. 1. The broadening of the individual spectra at temperatures of 54.1 and $69.2 \mathrm{~K}$ is apparent. The temperature dependent linewidths $\Gamma(T)$ of $S(Q, \omega)$ were obtained, as outlined in Sec. III C. From $\ln \Gamma(T)$ vs $1000 / T$ the activation energy is easily obtained from the slope of the fitted straight lines [Figs.
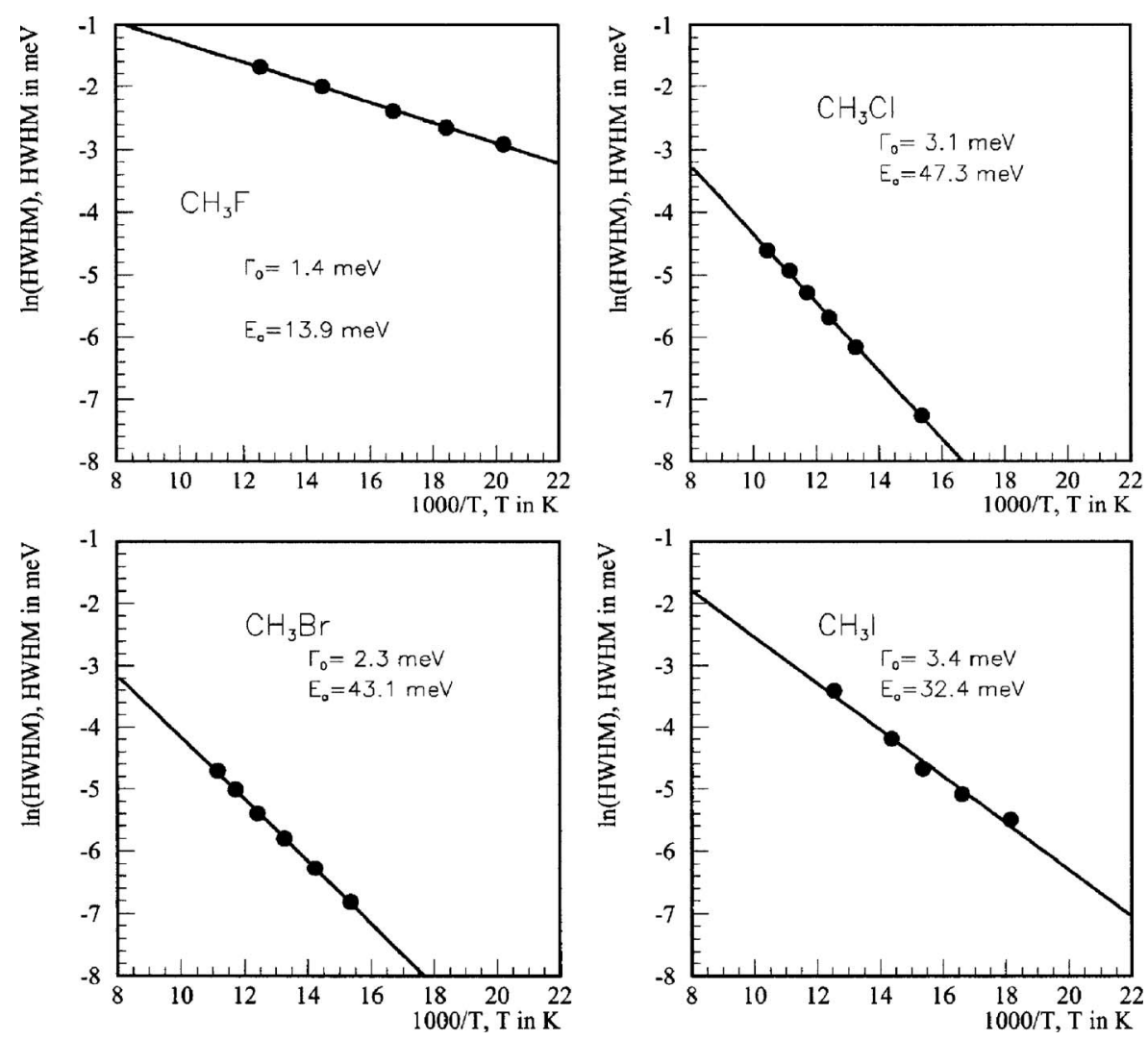

FIG. 2. Arrhenius plots of the temperature dependent linewidth of the individual methyl halides, which were obtained from the quasielastic neutron scattering experiments. 
TABLE I. Experimentally determined activation energies and attempt frequencies of the individual methyl halide.

\begin{tabular}{ccc}
\hline \hline Molecule & $\begin{array}{c}E_{a} \\
(\mathrm{meV})\end{array}$ & $\begin{array}{c}\Gamma_{0} \\
(\mathrm{meV})\end{array}$ \\
\hline $\mathrm{CH}_{3} \mathrm{~F}$ & $13.9( \pm 0.7)$ & $1.4( \pm 0.7)$ \\
$\mathrm{CH}_{3} \mathrm{Cl}$ & $47.3( \pm 1.5)$ & $3.1( \pm 0.7)$ \\
$\mathrm{CH}_{3} \mathrm{Br}$ & $43.1( \pm 0.9)$ & $2.3( \pm 0.3)$ \\
$\mathrm{CH}_{3} \mathrm{I}$ & $32.4( \pm 1.9)$ & $3.4( \pm 0.7)$ \\
\hline \hline
\end{tabular}

2(a)-2(d)]. The respective activation energies are shown in Table I together with the second fit parameter $\Gamma_{0}$. Values of $\Gamma_{0}$ for $\mathrm{CH}_{3} \mathrm{X}(\mathrm{X}=\mathrm{Cl}, \mathrm{Br}$, and $\mathrm{I})$ range between 2.3 and $3.4 \mathrm{meV}$ and are in reasonable agreement with typical attempt frequencies of methyl groups. The somewhat lower value of $1.4 \mathrm{meV}$ in the case of fluoride is atypical and will be addressed below. To obtain the activation energy for methyl iodide only the temperature range of $55 \leqslant T \leqslant 88 \mathrm{~K}$ was taken into account to ensure that the quasielastic linewidth is properly described by classical dynamics. ${ }^{1}$ Besides the activation energy the complete set of input parameters for the modeling included the tunneling transition as well as the first librational transition; values were taken from Refs. 1 and 2.

\section{B. Single particle model}

\section{1. $\mathrm{CH}_{3} \mathrm{~F}$ and $\mathrm{CH}_{3} \mathrm{l}$ : Assignments of the librational transition}

Previous inelastic neutron studies done on $\mathrm{CH}_{3} \mathrm{~F}$ (Ref. 2) were explained by applying the SPM model. The experimentally obtained values for the tunneling transition of $23.1 \mu \mathrm{eV}$ combined with a librational transition of $10 \mathrm{meV}$ can easily be reproduced with $\mathrm{meV}$ and $\alpha_{3}=0^{\circ}$. In this particular case we fit three potential parameters by using three experimentally determined transition energies in order to minimize $\chi_{\exp }^{2}$. The results of the model calculations are shown in Table II. Based on the values of $\chi_{\exp }^{2}$ we conclude that the SPM is not a suitable model to accurately describe the dynamics of fluoride and chloride. The SPM, however, seems to be the appropriate model in the case of bromide. For the given set of potential parameters the experimentally observed transitions and the calculated ones agree quite well.

\section{2. $\mathrm{CH}_{3} \mathrm{I}$}

To a certain extent there is a good agreement in the case of iodide but only under the assumption that the $R_{z}$ libration

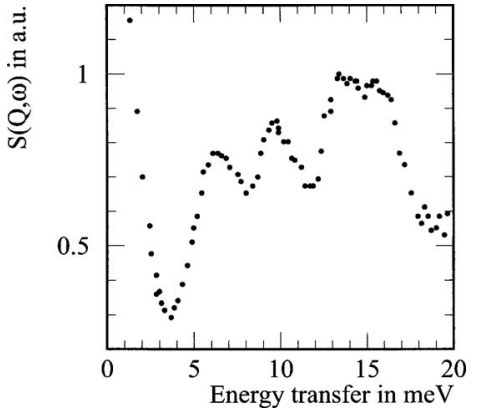

FIG. 3. DOS of $\mathrm{CH}_{3} \mathrm{~F}$ (Ref. 2). The librational transition is assigned to the peak at $10 \mathrm{meV}$. Coupling between two in-plane methyl groups may cause an intensity between 16 and $18 \mathrm{meV}$.

is in the order of $14 \mathrm{meV}$ rather than $13 \mathrm{meV}$, which would endorse the Raman assignment made in Ref. 19. The authors in Ref. 1 used the isotope effect to identify and distinguish between $R_{x}, R_{y}$, and $R_{z}$ modes and concluded that the peak at $14.57 \mathrm{meV}$ represents a $R_{x}$ and $R_{y}$ modes since it scales almost perfectly with $\left(m_{1} / m_{2}\right)^{1 / 2}$, with $m_{1}=18 m_{H}$ being the mass of the deuterated and $m_{2}=15 m_{H}$ the mass of the hydrogenated methyl group. The $14.57 \mathrm{meV}$ line shifts to $13.1 \mathrm{meV}$ at $T=18 \mathrm{~K}(12.7 \mathrm{meV}$ at $T=60 \mathrm{~K})$ and agrees well with the Raman assignments of 12.76 and $12.95 \mathrm{meV}$ at $T=20 \mathrm{~K}$. The peak at $13.27 \mathrm{meV}$, identified to be an $R_{z}$ mode, should show in harmonic approximation an isotope shift of $\left(B_{H} / B_{D}\right)^{1 / 2}$ with $B_{i}$ being the rotational constants of the hydrogenated and deuterated methyl group. Therefore the line should have shifted by a factor of $\sim \sqrt{2}$ to $9.4 \mathrm{meV}$. By chance this is also the isotope effect if the Schrödinger equation is solved with the rotational constant $B_{D}$ of the deuterated a threefold potential of $23.7 \mathrm{meV}$. The assignment of the librational transition energy of $10 \mathrm{meV}$ to the corresponding peak at $10.1 \mathrm{meV}$ in the density of states (DOS) was done via the temperature dependent line broadening of the tunneling peak despite the fact that this peak did not show the strongest amplitude (Fig. 3). Looking at the DOS of $\mathrm{CH}_{3} \mathrm{~F}$ there may be some ambiguity with regard to the assignment of peaks to the methyl librations but a librational transition of $7 \mathrm{meV}$, as suggested by $\mathrm{NMR}-\mathrm{T}_{1}-\mathrm{M}_{2}$ and Raman-IR experiments, was disproved on the basis of a wrongly applied formula and the isotope effect. For $\mathrm{CH}_{3} \mathrm{I}$, the authors in Ref. 1 used the isotope effect to assign the peak at $13.27 \mathrm{meV}$ as the librational mode $\left(R_{z}\right.$ libration in Ref. 1), which identified this mode as a methyl group rotation around the $\mathrm{C}-\mathrm{I}$ bond and the peak at $14.54 \mathrm{meV}$ as a

TABLE II. Potential parameters and characteristic transitions calculated for the methyl halides assuming the SPM. Experimentally determined transition energies and derived potential parameters are compared to SPM fits. Input parameters are taken from Refs. 1 and 2. Units are $\mu \mathrm{eV}$ for $\hbar \omega_{t}, \mathrm{meV}$ for $E_{01}, E_{a}, V_{3}$, and $V_{6}$, and degrees for $\alpha_{6}$.

\begin{tabular}{lcccc}
\hline \hline Molecule & $\begin{array}{c}\text { Experimental input parameters } \\
\hbar \omega_{t}, E_{01}, E_{a}\end{array}$ & $\begin{array}{c}\mathrm{SPM} \text { potential parameters } \\
V_{3}, V_{6}, \alpha_{6}\end{array}$ & $\begin{array}{c}\text { Calculated transitions } \\
\hbar \omega_{t}, E_{01}, E_{a}\end{array}$ & $\chi_{\exp }^{2}$ \\
\hline $\mathrm{CH}_{3} \mathrm{~F}$ & $23.1,10.0,13.9$ & $22.8,2.1,143.3$ & $22.30,10.6,16.7$ & 0.525 \\
$\mathrm{CH}_{3} \mathrm{Cl}$ & $0.1,18.1,47.3$ & $59.1,7.8,111.0$ & $0.26,19.8 .48 .3$ & 0.270 \\
$\mathrm{CH}_{3} \mathrm{Br}$ & $0.9,15.8,43.1$ & $52.3,-0.2,143.4$ & $0.92,15.8,43.8$ & 0.011 \\
$\mathrm{CH}_{3} \mathrm{I}$ & $2.44,13.27,32.4$ & $41.96,1.27,2.1$ & $2.36,14.5,34.1$ & 0.201 \\
& $2.44,14.19,32.4$ & $40.9,2.1,32.4$ & $2.46,14.7,33.0$ & 0.026 \\
\hline \hline
\end{tabular}


TABLE III. Potential parameters and characteristic transitions calculated for the methyl halides assuming the CM. Experimentally determined energy transitions and derived potential parameters are compared to calculations. Input parameters are taken from Refs. 1 and 2 . Units are $\mu \mathrm{eV}$ for $\hbar \omega_{t}$, and $\delta \hbar \omega_{t}$ and $\mathrm{meV}$ for $E_{01}, E_{a}, V_{3}$, and $W_{3}$.

\begin{tabular}{cccccc}
\hline \hline & $\begin{array}{c}\text { Experimental input parameters } \\
\hbar \omega_{t}, E_{01}, E_{a}\end{array}$ & $\begin{array}{c}\mathrm{CM} \text { potential parameters } \\
V_{3}, W_{3}\end{array}$ & $\begin{array}{c}\text { Calculated transitions } \\
\hbar \omega_{t}, \delta \hbar \omega_{t}, E_{01}, E_{a}\end{array}$ & $\chi_{\exp }^{2}$ & $Q\left(\chi_{\exp }^{2}, 1\right)$ \\
\hline $\mathrm{CH}_{3} \mathrm{~F}$ & $23.1,10.0,13.9$ & $20.2,-2.4$ & $23.14,0.12,10.1,13.9$ & 0.002 \\
$\mathrm{CH}_{3} \mathrm{Cl}$ & $0.1,18.1,47.3$ & $71.9,3.5$ & $0.25,0.0,18.1,46.1$ & 0.121 & 0.964 \\
$\mathrm{CH}_{3} \mathrm{Br}$ & $0.9,15.8,43.1$ & $53.0,0.4$ & $0.90,0.0,15.8,35.4$ & 1.699 & 0.714 \\
$\mathrm{CH}_{3} \mathrm{I}$ & $2.44,13.27,32.4$ & $48.1,3.3$ & $2.44,0.0,13.3,26.5$ & 1.314 \\
& $2.44,14.19,32.4$ & $42.3,-1.0$ & $2.12,0.0,14.4,28.9$ & 0.474 \\
\hline \hline
\end{tabular}

motion perpendicular to the $\mathrm{C}-\mathrm{I}$ bond $\left(R_{x}\right.$ and $R_{y}$ in Ref. 1$)$. Raman experiments done on the methyl halides ${ }^{19}$ suggest, however, that the $R_{z}$ libration is $14.19 \mathrm{meV}$, whereas the $R_{x}$ and $R_{y}$ motions are transitions at 13.26 and $13.44 \mathrm{meV}$. This issue will be addressed in the following together with the assignment of the $10 \mathrm{meV}$ transition of $\mathrm{CH}_{3} \mathrm{~F}$.

\section{Results $\mathrm{CH}_{3} \mathrm{~F}, \mathrm{CH}_{3} \mathrm{Cl}$, and $\mathrm{CH}_{3} \mathrm{Br}$}

By assuming that the SPM is the appropriate model to describe the dynamics of the rotating methyl group the experimental parameters obtained for each individual halide allow us to determine three parameters of the rotational potential $V(\varphi)$, as given by Eq. (1). Without restricting the generality we set $V_{0}=0$ methyl group under otherwise identical conditions. This coincidence was taken as an argument in favor of the proposed mode assignment. In summary this interpretation shows the right isotope effect for the tilt but a somewhat strong isotope effect of the libration.

An inverted assignment is also possible. While there is the problem that the tilt mode is almost not influenced by deuteration it allows a perfect description of methyl libration. With SPM parameters from Table II, $V_{3}=40.9 \mathrm{meV}, V_{6}$ $=2.1 \mathrm{meV}$, and $\alpha_{6}=32.4^{\circ}$, the hydrogenated sample has $\hbar \omega_{t}^{H}=2.46 \mu \mathrm{eV}, E_{01}^{H}=14.67 \mathrm{meV}$, and $E_{a}^{H}=32.98 \mathrm{meV}$, and the Deuterated sample has $\hbar \omega_{t}^{D}=0.03 \mu \mathrm{eV}, E_{01}^{D}$ $=10.80 \mathrm{meV}$ (transition observed at $10.6 \mathrm{meV}$ in the experiment).

This potential also fits the newly observed activation energy and is consistent with the earlier Raman assignment. Based on this result we identify the experimentally determined transition at $14.57 \mathrm{meV}$ as the $R_{z}$ mode. Of course, the assumption in all this modeling is that all modes have Einstein character. The neutron experiment, however, samples the first Brillouin zone as a whole. Since the tilt and librational modes are energetically very close it is possible that the mode character changes along a dispersion branch due to coupling [as indicated in Fig. 5d in Ref. 9]. Since dispersion is not contained in the SPM the calculated transition can only define a mean librational transition. Such effect is clearly observed in dimehtylacetylene (DMA) ${ }^{20}$ where strong dispersion of librational modes resulted in two distinct and well separated peaks in the DOS. Secondly, the unusual temperature evolution of the tunneling transition in methyl iodide could be explained by such coupling of methyl rotation to phonons. Raman experiments, on the other hand, determine transitions at the $\Gamma$ point $(Q=0)$. This different weighting may easily lead to different observed eigenenergies.

\section{Coupling model}

Contrary to the procedure assuming the SPM, we have three observables and two parameters to fit. Table III shows the calculated values for the threefold part of the rotational potential and the coupling term and compares the obtained transitions with experimental values for the individual halide.

\section{1. $\mathrm{CH}_{3} \mathrm{~F}$}

The tunneling transition of $\mathrm{CH}_{3} \mathrm{~F}$ were obtained on IN10 at the ILL with an energy resolution of $1 \mu \mathrm{eV}{ }^{2}$ The author mentioned that the low temperature peak of the tunneling transition can be well described by the resolution function implying that the coupling between methyl groups was either weak or negligible. However, due to the smaller halide atom individual molecules must be closer together as compared to the other halides but a detailed discussion was not possible due to the unknown crystal structure of $\mathrm{CH}_{3} \mathrm{~F}$. For the given combination of $\left(V_{3}, W_{3}\right)=(20.2 \mathrm{meV},-2.4 \mathrm{meV})$ we obtain transitions that are shown in Table IV. The averaged tunneling energy is almost perfectly reproduced and the split of the tunneling sublevels due to the coupled motion is $0.12 \mu \mathrm{eV}$. The mean librational transition is calculated to be $E_{01}$ $=10.1 \mathrm{meV}$ and in full agreement with experiments and so is the activation energy of $E_{a}=13.7 \mathrm{meV}$. Also, the more complicated transition scheme is backed up by the DOS, which does not show one distinct peak but a more complex spectrum. The split of the tunneling sublevels could potentially be determined experimentally by using an inelastic neutron scattering instrument such as IN10 with an energy resolution of $\delta E=0.3 \mu \mathrm{eV}$ [e.g., the SPHERES spectrometer at FRM-II with the polished $\mathrm{Si}(111)$ analyzer-monochromator setup at a neutron wavelength of $6.27 \AA]$ as the splitting would be in

TABLE IV. Calculated transition between the ground state sublevels for the experimental tunneling peak at higher energy transfer for $\mathrm{CH}_{3} \mathrm{~F}$.

\begin{tabular}{cc}
\hline \hline Symmetry & Transition $[\mu \mathrm{eV}]$ \\
\hline$A A \rightarrow A E$ & 23.14 \\
$A A \rightarrow E_{a} E_{b}$ & 23.00 \\
$A A \rightarrow E_{a} E_{a}$ & 23.02 \\
$E_{a} E_{b} \rightarrow E_{a} E_{a}$ & 0.02 \\
\hline
\end{tabular}




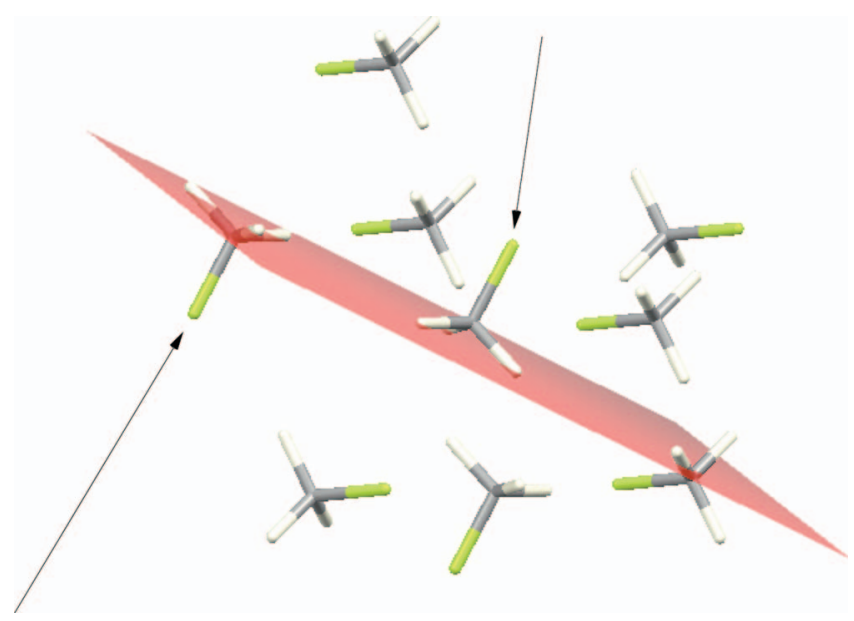

FIG. 4. (Color) Plane (gray shaded) shared by two methyl groups (indicated by arrows) necessary to apply the coupling model. The plane shown is parallel to the crystallographic $(14 \overline{1})$ plane.

the order of $30 \%$ of the resolution of the instrument. It is clear, however, that it was not possible to observe a possible line broadening earlier simply because of the limited energy resolution. Based on $\chi_{\exp }^{2}$ and $Q\left(\chi_{\exp }^{2}, 1\right)$ we conclude that the $\mathrm{CM}$ is the most likely model to explain the dynamics of the methyl fluoride.

\section{2. $\mathrm{CH}_{3} \mathrm{~F}$ Mode damping}

As mentioned previously and described in Ref. 2 the DOS of $\mathrm{CH}_{3} \mathrm{~F}$ (shown in Fig. 3) is somewhat unusual in the sense that the libration of the methyl group around its bond axis did not show the strongest amplitude, which would normally be expected due to the large incoherent scattering cross section of the hydrogen and the large displacements of librons in the meV range. ${ }^{21}$ The damped intensity of the librational peak at $10 \mathrm{meV}$ was explained by assuming large displacements $R_{x y}$ perpendicular to $R_{z}$ in combination with a short $\mathrm{C}-\mathrm{F}$ bond distance and less molecular asymmetry as compared to the other halides. Punkinnen, ${ }^{22}$ for example, assumed that the reorientation rate $1 / \tau \propto \Gamma$ would be proportional to the libration amplitude $\left\langle\varphi^{2}\right\rangle$ of the methyl group and eventually derived a formula similar to Eq. (7) with the librational transition $E_{01}$ rather than $E_{a}$. In both cases, however, the prefactor gives an estimate of the attempt frequency or reorientation rate. The scattering function and linewidth, as shown in Eqs. (6) and (7) imply reorientations via the bond axis. A somewhat lower value of $\Gamma_{0}=1.4 \mathrm{meV}$ for the methyl fluoride, as shown in Table I, means a lower reorientation rate and would indicate that the amplitudes of the $R_{z}$ librations are indeed suppressed due to larger $R_{x y}$ amplitudes as suggested earlier.

For fluoride the CM shows that the coupling is roughly ten times weaker than the dominant threefold part of the rotational potential. Of course, the model requires methyl groups that share a common plane. The recently determined crystal structure of methyl fluoride ${ }^{7}$ allows us to look for such $\mathrm{CH}_{3}$ groups and, as indicated in Fig. 4, such a plane would be parallel to the crystallographic $(14 \overline{1})$ plane with an in-plane $\mathrm{H}-\mathrm{H}$ distance of different methyl groups of

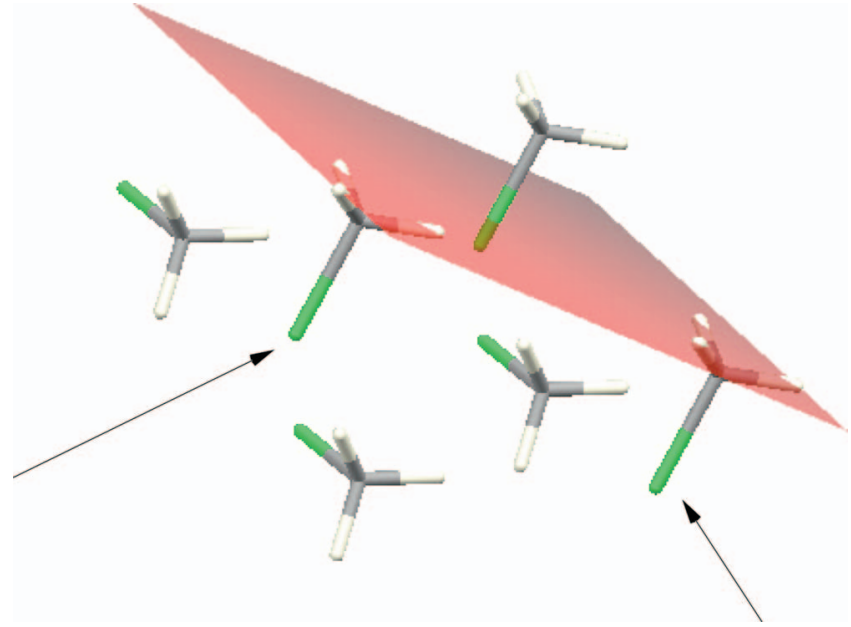

FIG. 5. (Color) Crystallographic plane (gray shaded) shared by two methyl groups (indicated by arrows) of $\mathrm{CH}_{3} \mathrm{Cl}$ necessary to apply the coupling model.

$3.032 \AA$ A. Similar to discussions in Ref. 23 , a more complex transition scheme combined with dispersion could result in less pronounced librational peaks, and a further suppression of intensity could occur via the above-mentioned damping. The coupling model also yields transitions in the range of $16-18 \mathrm{meV}$, and these additional features as well as the magnitude of the splitting would not contradict the experimental DOS (Fig. 3) nor the calculated DOS in Ref. 9. Additionally, phase angles should not occur due to crystallographic symmetry and the relative orientation of the in-plane methyl groups, which is also indicated in Fig. 4.

\section{3. $\mathrm{CH}_{3} \mathrm{Cl}$ Coupling versus single particle model}

By assuming coupling between methyl groups the dynamics of the $\mathrm{CH}_{3} \mathrm{Cl}$ is also better reproduced as compared to the SPM. A crystallographic plane in which methyl groups lie that shows coupled motions is shown in Fig. 5. The shortest in-plane $\mathrm{H}-\mathrm{H}$ distance of different methyl groups is $4.715 \AA$ A resulting in a less pronounced coupling compared to the fluoride. In fact, the term $W_{3}$ is roughly 20 times lower than the actual value of $V_{3}$ and the coupling, therefore, is a factor of 2 weaker as compared to the fluoride. The quality of the fit is not as good as for the fluoride, but this could primarily be due to the experimental tunneling transition of $0.1 \mu \mathrm{eV}$. This value is much lower than the energy resolution of the spectrometer used during the experiment and not a "good" input parameter as it is affected by a significant uncertainty. Interestingly, not only the CM but also the SPM calculations find tunneling transitions in the order of $\sim 0.25 \mu \mathrm{eV}$.

\section{4. $\mathrm{CH}_{3} \mathrm{Br}$ and $\mathrm{CH}_{3} \mathrm{I}$}

In the remaining two halides the in-plane next-group distances increase up to $\sim 6 \AA$ making coupling less likely. In addition, the experimental transitions cannot be reproduced by the model calculations, and based on the values for $\chi_{\exp }^{2}$ and $Q\left(\chi_{\exp }^{2}, 1\right)$, we conclude that the CM is not the suitable model to describe the dynamics of bromide and iodide. 


\section{SUMMARY}

Quasielastic neutron scattering experiments were carried out using the NEAT spectrometer at the HMI and the BSJ spectrometer at the FZJ. Activation energies for $\mathrm{CH}_{3} \mathrm{~F},-\mathrm{Cl}$, $-\mathrm{Br}$, and $-\mathrm{I}$ were obtained. The combination of tunneling, librational, and activation energies allowed us to determine potential parameters that describe the dynamics of the methyl groups. Assuming the SPM there is a good agreement between the experiment and the model for methyl bromide and iodide. For the latter, however, the SPM is applicable only if we reassign the librational transition to be $14.54 \mathrm{meV}$ rather than $13.27 \mathrm{meV}$ thus endorsing earlier Raman experiments.

In the cases of fluoride and chloride the observed dynamics are better described by the coupling model. There is a good agreement between experimental and calculated transitions, and the crystal structure of the individual molecules provides the lattice planes required for applying the $\mathrm{CM}$. The coupling gets less strong with the increasing size of the halide making it the less favorable model for $\mathrm{CH}_{3} \mathrm{Br}$ and $\mathrm{CH}_{3} \mathrm{I}$.

\section{ACKNOWLEDGMENTS}

The authors would like to thank the BENSC and FZJ for the beam time and the excellent support during the experiments. One of the authors (O.K.) would like to acknowledge the funding through Access to Major Facilities Program under Program Nos. 03/04-N-12 and 04/05-N-30 for the financial support. He would also like to thank Dr. M. James for his interest and the discussions related to this paper.
${ }^{1}$ M. Prager, J. Stanislawski, and W. Häussler, J. Chem. Phys. 86, 2563 (1987).

${ }^{2}$ M. Prager, J. Chem. Phys. 89, 1181 (1988).

${ }^{3}$ P. N. Gerlach, B. H. Torrie, and B. M. Powell, Mol. Phys. 57, 919 (1986).

${ }^{4}$ R. D. Burbank, J. Am. Chem. Soc. 75, 1211 (1953).

${ }^{5}$ M. Kawaguchi, M. Hijikigawa, Y. Hayafuji, M. Ikeda, R. Fukushima, and Y. Tomie, Bull. Chem. Soc. Jpn. 46, 53 (1973).

${ }^{6}$ R. M. Ibberson and M. Prager (unpublished).

${ }^{7}$ R. M. Ibberson and M. Prager, Acta Crystallogr., Sect. B: Struct. Sci. 52, 892 (1996).

${ }^{8}$ W. Press, Single Particle Rotations in Molecular Crystals, Springer Tracts in Modern Physics Vol. 92 (Springer, Berlin, 1981).

${ }^{9}$ O. Kirstein and M. Prager, J. Chem. Phys. 120, 5199 (2004).

${ }^{10}$ A. K. Rappé, C. J. Casewitt, K. S. Colwell, W. A. Goddard III, and W. M. Skiff, J. Am. Chem. Soc. 114, (1992).

${ }^{11}$ W. Häusler and A. Hüller, Z. Phys. B: Condens. Matter 59, 177 (1985).

${ }^{12}$ M. Beé, Quasielastic Neutron Scattering (Hilger, Bristol, 1998).

${ }^{13}$ www.hmi.de/bensc/instrumentation/instrumente/v3/v3_en.htm

${ }^{14}$ Neutron Scattering and Experiments at the Research Reactor in Jülich (Forschungszentrum Jülich, Jülich, 2003), p. 34.

${ }^{15}$ TOFSYs is developed and maintained by the Institut für Festkörperforschung, Forschungszentrum Jülich GmbH, Germany.

${ }^{16}$ R. Zorn, B. Frick, and L. Fetters, J. Chem. Phys. 116, 845 (2002).

${ }^{17}$ D501, and D507, CERN program library (www.cernlib.ch).

${ }^{18}$ I. N. Bronstein and K. A. Semendjajew, Taschenbuch der Mathematik (Deutscher Verlag, Berlin, 1987) Secs. 1.1.2.10 and 5.2.2.3.

${ }^{19}$ O. S. Binbrek, A. Anderson, B. Andrews, and B. H. Torrie, J. Raman Spectrosc. 15, 406 (1984).

${ }^{20}$ O. Kirstein, M. Prager, M. R. Johnson, and S. F. Parker, J. Chem. Phys. 117, 1313 (2002).

${ }^{21}$ A. C. Zemach and R. J. Glauber, Phys. Rev. 101, 118 (1956).

${ }^{22}$ M. Punkinnen, Phys. Rev. B 21, 54 (1980).

${ }^{23}$ O. Kirstein, M. Prager, R. M. Dimeo, and A. Desmedt, J. Chem. Phys. 122, 14501 (2005). 